\title{
RORgamma Agonist LYC-55716
}

National Cancer Institute

\section{Source}

National Cancer Institute. RORgamma Agonist LYC-55716. NCI Thesaurus. Code C131294.

An orally bioavailable agonist of retinoic acid-related orphan receptor gamma (RORg), with potential immunomodulatory and antineoplastic activities. Upon oral administration of RORg agonist LYC-55716, this agent selectively binds to the nuclear receptor transcription factor RORg, forming a receptor complex that translocates to the nucleus, and binds to ROR response elements (ROREs), enhancing the function, proliferation and survival of type 17 T-cells, including Th17 (helper T-cells) and Tc17 (cytotoxic T-cells). This may increase the expression of co-stimulatory molecules and decrease the expression of co-inhibitory molecules on T-cells leading to increased production of cytokines and chemokines by T-cells, decreased proliferation of regulatory T-cells (Tregs), and abrog ation of tumor-induced immunosuppression. This ultimately induces a T-cell-mediated immune response against cancer cells and leads to a reduction in tumor cell growth. RORg, the nuclear receptor transcription factor that is involved in Th17/T c17 differentiation, plays a key role in immune activation. 